\title{
Norois
}

Environnement, aménagement, société

\section{Rapports multisensoriels des habitants à leurs territoires de vie. Retours critiques sur une démarche méthodologique}

Inhabitant's multisensory relations with their territories. Critical feedback on a methodological approach

\section{Théa Manola}

\section{OpenEdition \\ Journals}

Édition électronique

URL : http://journals.openedition.org/norois/4649

DOI : $10.4000 /$ norois.4649

ISBN : 978-2-7535-2916-8

ISSN : 1760-8546

Éditeur

Presses universitaires de Rennes

\section{Édition imprimée}

Date de publication : 30 juin 2013

Pagination : 25-42

ISBN : 978-2-7535-2882-6

ISSN : 0029-182X

Référence électronique

Théa Manola, «Rapports multisensoriels des habitants à leurs territoires de vie. Retours critiques sur une démarche méthodologique », Norois [En ligne], 227 | 2013, mis en ligne le 30 juin 2015, consulté le 30 avril 2019. URL : http://journals.openedition.org/norois/4649; DOI : 10.4000/norois.4649 


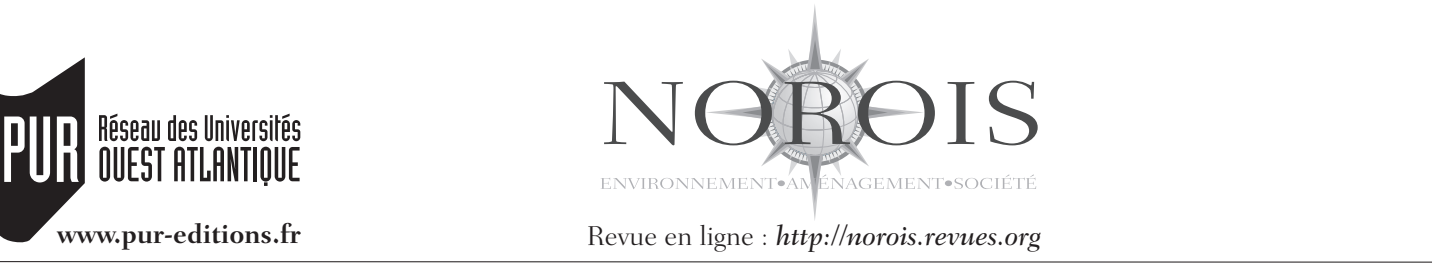

\title{
Rapports multisensoriels des habitants à leurs territoires de vie Retours critiques sur une démarche méthodologique
}

\author{
Inhabitant's Multisensory Relations with their Territories \\ Critical Feedback on a Methodological Approach
}

\begin{abstract}
Théa ManOLA
Lab’Urba, Institut d'Urbanisme de Paris, UPEC, 61 av. du Général-de-Gaulle - 94100 Créteil; Atelier de Recherche Politopie, 111 rue Gabriel-Péri-94400 Vitry-Sun-SEine (thea.manola@gmail.com)
\end{abstract}

\begin{abstract}
Résumé : La prise en compte des sens, autres que la vue, dans l'analyse des espaces urbains constitue une question peu traitée. Si une production scientifique importante existe sur les rapports sensoriels que les hommes entretiennent avec leurs territoires de vie, une réelle difficulté persiste quant à leur considération dans l'action urbaine. Une des raisons de cette difficulté relève des faibles moyens dont nous disposons pour les appréhender. Comment surmonter la difficulté des individus à faire part de leur expérience sensible et les amener à parler à ce sujet? Cet article présente, de manière critique, la démarche méthodologique emboîtée mise en place dans le cadre de notre recherche doctorale ainsi que d'une recherche menée dans le cadre du PIRVE-2008 financée par le PUCA et le CNRS. Cette démarche méthodologique, mise en œuvre auprès des habitants de trois quartiers « durables », combine des entretiens ouverts courts, des parcours multisensoriels et des «baluchons multisensoriels », afin de pallier aux difficultés relatives à l'expression du sensible. Il présente alors les principaux résultats méthodologiques de ce travail : évolutions et adaptations du dispositif; apports et complémentarité des différents outils méthodologiques, ainsi que les retours critiques sur les baluchons multisensoriels, outil nouvellement créé et testé.
\end{abstract}

\begin{abstract}
The senses, other than sight, and their inclusion in the urban space are a neglected issue. If there is an important scientific production on the sensory human relations, a real difficulty exists in terms of consideration in urban projects of this question. One reason for this difficulty is related to means that we have to help us understand those sensory relations. How can we get people to talk about their sensory and sensible relations with their environment, when we know that many difficulties exist regarding their expression? This paper presents, with a critical point of view, the methodology, established in the framework of our PhD research as well as research conducted in PIRVE-2008 funded by the PUCA and CNRS. This methodology combines: brief open-ended interviews, "multisensory walks" and "multisensory pouches" (methods elaborated specifically for this research) carried out with the residents of the three so-called sustainable neighborhoods and that was thought as a way to overcome the difficulties in expression of the "sensible" relations. This paper presents the main methodological results of this research: evolution and adaptation of the methodology; results of each method and complementarities between them; success and specificities of "multisensory pouches".
\end{abstract}

Mots clés : Sensible - paysage multisensoriel - innovation méthodologique - parcours - baluchons sensoriels

Keywords: multi-sensory landscape - methodological innovation - multisensory walks - multisensory pouches 


\section{INTRODUCTION}

L'intérêt grandissant pour la question du paysage est à ce jour une réalité (Blanc et al., 2004) et fait écho à des changements, actuellement en cours, dans la théorie du paysage (Bedard, 2009). Le paysage est considéré dans ce cadre comme un rapport entre l'expérience sensible des habitants et les territoires de vie, rapport dont l'analyse fait appel à des éléments de connaissance et de débat relevant, certes, de la dimension physique du milieu mais aussi du sensible et du symbolique (Berque, 2000; Besse, 2009). Les réglementations internationales, telle que la Convention Européenne du Paysage adoptée à Florence en 2000, mais aussi l'« irrésistible ascension des paysagistes »(Marot, 1995) témoignent de ce retour en force.

En parallèle, on constate l'évolution d'une demande sociale de paysage (Luginbühl, 2001) issue notamment du désir d'une qualité du cadre de vie, d'un «mieux vivre », voire d'un « bien-être », qui font de plus en plus l'objet d'une sensibilité particulière de la part des populations. Certaines publications « grand public » sur la thématique paysagère, comme l'article de De Jarcy et de Rem ${ }^{1}$, témoignent de cette sensibilité.

Dans ce contexte, le paysage apparaît comme une préoccupation récurrente et fait écho à la montée en puissance des problématiques environnementales et du développement durable.

Dans le même temps et dans le prolongement des réflexions des dernières décennies, la question des rapports sensoriels à la ville commence à être de plus en plus traitée, mais de manière confinée au sein de certaines disciplines (ex. anthropologie) et laboratoires (ex. UMR Ambiances, Lab'urba, UMR ESO). Pourtant, la question sensorielle et, plus largement, du sensible, fait de plus en plus parler d'elle aussi bien dans la recherche en sciences humaines et sociales que dans les métiers de la conception architecturale et urbaine comme le montrent la création de réseaux scientifiques ${ }^{2}$ et la multiplication de colloques sur la question des sens en ville ${ }^{3}$. D'autre part, le nombre de publications scientifiques

1. Jarcy (de) X. et Remy V., 2010. Comment la France est devenue moche, Enquête Télérama, n 3135, cf. [http://wwww.telerama.fr/monde/commentla-france-est-devenue-moche, 52457.php].

2. Par exemple «Ambiances.net »

3. Par exemple « Sentir et ressentir la ville», Lisbonne, 2010 ; « Les sens en ville du Moyen-Age à nos jours », Tours, 2010. dédiées à la question ne cesse d'augmenter (Paquot, 2010) ${ }^{4}$. Enfin, apparaissent timidement des projets urbains avec une entrée sensorielle - à l'instar du lot 5 « La Défense des sens »- et émerge une médiatisation de projets architecturaux traitant des sens, comme en témoigne le Pavillon français de l'exposition universelle de 2010.

Si des travaux existent sur le sensible et si, dans la théorie du paysage, la multisensorialité de ce dernier semble acquise (Luginbühl, 2005), peu de recherches empiriques et relatives à l'espace urbain traitent directement de «paysage multisensoriel ». Malgré les travaux plus ou moins anciens sur les paysages monosensoriels (Balez, 2000; Grésillon, 2010) et les réflexions théoriques, philosophiques ou poétiques qui traitent de la multisensorialité spatiale (Corbin, 1986; Sansot, 2004), le paysage multisensoriel constitue un point faible de la connaissance empirique et se trouve peu considéré dans l'action urbaine. Ce faisant, les « sentir » et « ressentir » des habitants, mais aussi leurs expériences et leurs capacités d'action, sont partiellement pris en compte dans les procédures souvent formalisées d'une démocratie qui se veut pourtant plus participative (Callon, Lascoumes, Barthes, 2001). En conséquence, les actions paysagères et urbanistiques sont souvent pensées en des termes formels voire normatifs, souvent esthétiques et patrimoniaux, guidées par la primauté de la vue. En conséquence, le rapport sensible 5 au Monde, par le biais privilégié que nous considérons ici - le paysage - est difficile à prendre en compte dans une visée opérationnelle.

Comment saisir alors les rapports multisensoriels que les habitants entretiennent avec leurs territoires de vie? Comment prendre en compte le sensible dans l'action urbaine? Ces interrogations se heurtent à plusieurs verrous en lien avec les champs de l'action : référentiels, habitudes historiques des métiers de la conception, prépondérance d'une pensée cartésienne, etc. Mais, d'autres raisons existent, au croisement cette fois de l'action et des habitants : les questions méthodologiques.

\footnotetext{
4. Pour les revues, voir : Place publique, $\mathrm{n}^{\circ} 22,2010$; Urbanisme, $\mathrm{n}^{\circ} 34$, 2008.

5. Le sensible intègre les significations des rapports sensoriels. Nous utilisons le terme sensible car il semble englober tous les problèmes d'ordre méthodologique auxquels notre problématique peut se confronter : aborder les sens mais aussi leurs significations.
} 
Les questions méthodologiques, sur lesquelles est centrée cette contribution, constituent un sujet de réflexion en soi et, selon nous, pour deux raisons. La première est que, si "les valeurs des paysages reposent davantage sur une variété de facteurs liés aux émotions, au vécu et aux connaissances que sur la considération exclusive de paramètres visuels formels » (Vouligny et Domon, 2006, p. 1), il n'est pas moins vrai que nous peinons encore à ce jour à saisir le «sentir » et « ressentir» des habitants d'un point de vue méthodologique. D’autre part, les questions méthodologiques sont des conditions opératoires de prise en compte du sensible sur lesquelles les chercheurs mais aussi les acteurs opérationnels se penchent. De ce fait, elles semblent être un des terrains d'échange potentiel entre eux.

Cet article vise à interroger les possibles méthodes pour permettre une approche sensible de l'espace par l'action urbaine, et ce, via le paysage multisensoriel. Pour ce faire, nous nous appuierons sur le retour d'expérience d'une première enquête (Faburel et Manola, 2007), et surtout sur les résultats de notre travail doctoral (Manola, 2012) et d'une recherche menée dans le cadre du PIRVE2008 financée par le PUCA et le CNRS (Faburel et al., 2011). De ces deux derniers travaux, seront ici mis en avant les résultats d'une démarche méthodologique emboîtée et pour partie innovante, mise en œuvre sur trois quartiers dits «durables »: Wilhelmina Gasthuis Terrein (WGT) à Amsterdam (Pays-Bas), Augustenborg à Malmö (Suède), Bo01 à Malmö (Suède).

Nous présenterons, dans un premier temps, les difficultés quant à la saisie et la compréhension de l'expérience sensible des habitants, la démarche méthodologique adoptée au regard de nos objectifs, ainsi que les trois quartiers durables d'application choisis. Puis, nous aborderons les ajustements/évolutions méthodologiques apportés au cours de la mise à l'épreuve du terrain, afin de garantir l'apport des méthodes emboîtées, notamment au regard de l'introduction des «baluchons multisensoriels». Enfin, nous dresserons un premier bilan des enseignements de notre démarche méthodologique pour en proposer d'ores et déjà des évolutions et/ou des améliorations possibles.

\section{UNE DÉMARCHE MÉTHODOLOGIQUE POUR MIEUX PRENDRE EN COMPTE LES RAPPORTS MULTISENSORIELS AU TERRITOIRE DES HABITANTS}

\section{Quelques difficultés méthodologiques persistantes}

Un premier problème est prégnant dans notre travail : celui de la communication des expériences sensibles. En effet, suite à des entretiens exploratoires menés avec des habitants (Faburel et Manola, 2007), il est apparu que les enquêtés éprouvaient, pour certains, de grandes difficultés, pour d'autres, de la gêne à parler du sensible, de leurs sensations et de leurs expériences. Il est vrai que, comme bien d'autres ont pu le montrer (Thibaud et al., 1998; Balez, 2000; Grosjean et Thibaud, 2001; Blanc et al., 2004; Grésillon, 2010), parler du sensible revient à parler d'une intimité. La sollicitation du chercheur et, a fortiori, de l'enquêteur peut alors être interprétée comme une intrusion. Il en résulte une plus grande facilité à parler des expériences visuelles et, secondairement, sonores, plutôt que des autres. Parallèlement, on observe un repli sur des notions plus usuelles, donc a priori une certaine « pauvreté » des champs lexicaux, en même temps qu'une inclinaison à parler de ce qui est jugé négatif dans l'expérience sensible, comme les bruits, les mauvaises odeurs ou les désagréments visuels.

Les raisons de ces difficultés et gênes sont multiples. Révéler le sensible dans un monde qui freine son expression et exige un discours rationnel conduit à des discours convenus pour faire montre d'une certaine maîtrise de soi et du Monde. Dans cet esprit, les gens hésitent à livrer leurs expériences personnelles, considérées comme trop " subjectives ", sans apport véritable, pour donner à entendre une expression raisonnée des vécus. Aussi, la construction même de la langue française, dotée différemment en mots selon les sens évoqués, amène-t-elle une difficulté. Si en Occident, la vue, sens dominant, possède un lexique propre très développé, il est par exemple quasiment impossible à un locuteur non spécialiste de décrire ses sensations odorantes avec le vocabulaire courant (Dubois, 2006). De plus, certains termes récurrents de notre travail, et notamment les vocables «paysage » et « ambiance » (Manola, Geisler, 2012) ne sont pas forcément utili- 
sés par tous les habitants avec la même signification, ni de la même manière que par les scientifiques. Voyons alors dans quelle mesure notre travail, via un dispositif méthodologique renouvelé, propose un dépassement des difficultés liées à l'expression des expériences sensibles par les individus.

\section{Proposition d'un dispositif méthodologique innovant pour faire face aux difficultés}

Ces difficultés préalables considérées, l'édification d'un dispositif méthodologique se heurte à trois enjeux principaux, tels que :

- tenter de minimiser les inhibitions liées à l'expression du sensible;

- éviter de mobiliser uniquement des discours verbaux comme seul support d'expression des expériences sensibles, sans pour autant les exclure;

- utiliser un langage réapproprié, en se basant sur le(s) sens que les habitants lui donnent.

Au vu de ces éléments ainsi que d'autres réflexions (Audas et Martouzet, 2008), l'usage d'une seule méthode semble insuffisant pour traduire la multiplicité des rapports sensibles de l'homme avec son environnement. Au lieu de chercher LA méthode, il nous est paru plus judicieux et justifié d'opter pour une démarche méthodologique emboitée.

Dans cette perspective, nous avons puisé dans le gisement des méthodes dites qualitatives ${ }^{6}$ car cellesci se présentent comme plus aptes à traiter des rapports sensibles que les méthodes dites quantitatives. L'application de telles méthodes, à des fins d'action, reste peu courante même si quelques laboratoires ${ }^{7}$ et structures de recherche et/ou d'expérimentation ${ }^{8}$ les utilisent couramment.

Ainsi, pour lever ou contourner autant que possible les différentes difficultés évoquées, et conscients des limites propres à chacune des

6. Voici une liste non exhaustive de ces méthodes : l'observation; les récits et les entretiens (de différents types : courts/longs, directifs/non directifs, en face à face/de groupe, etc.), qui tendent vers une mise en langage des expériences sensibles; les processus délibératifs et autres ateliers, qui tendent vers une mise en discours collectif des vécus; les cartes mentales, qui tendent vers une mise en forme territorialisée des expériences, notamment sensibles; les parcours et itinéraires, qui saisissent les expériences sensibles sur le vif; mais aussi les diverses réactivations sensorielles, qui permettent de caractériser les modes d'appréhension du rapport sensible au monde, ainsi que les significations que leur prêtent les différents individus voire les groupes.

7. Citons par exemple l'UMR CITERES, l'UMR Ambiances ou encore le Lab'Urba.

8. Citons par exemple Polimoph et le pOlau. méthodes, nous avons cherché à compléter certaines d'entre elles par d'autres. D'autre part, nous avons combiné des méthodes issues des sciences humaines et sociales avec des méthodes propres aux métiers de la conception architecturale, paysagère et/ou urbaine. C'est pourquoi, la démarche adoptée peut être qualifiée d'emboîtée, articulant de manière séquencée et progressive plusieurs dispositifs. Il ne s'agissait en aucun cas de proposer une procédure stabilisée et formalisée, voire normalisée, pour la prise en compte des rapports multisensoriels, mais d'assumer nos incertitudes et de nous laisser guider par nos expérimentations en les adaptant progressivement.

Cette démarche emboitée est composée d'investigations de terrain de deux natures différentes, comme nous allons le voir plus avant : un diagnostic urbain et paysager enrichi de discours d'acteurs, et surtout des investigations auprès des habitants.

Premièrement, il s'est agi de réaliser un diagnostic urbain et paysager à l'aide de discours d'acteurs ou « experts » afin d'aider à la connaissance et à la compréhension des quartiers. Ce diagnostic se compose lui-même de trois approches : l'une bibliographique et documentaire; une autre qui se rapproche fortement des diagnostics faits par les concepteurs, avec des relevés et descriptions, des observations plus fines des quartiers; une autre enfin, plus sensible, mobilisant notre propre expérience comme moyen de compréhension et d'analyse de l'espace afin d'avoir une connaissance sensorielle et sensible propre à chacun des trois quartiers étudiés. Et, afin d'obtenir d'autres informations clefs, le diagnostic est complété par une série d'entretiens semi-directifs avec des acteurs impliqués dans le conseil, la conception, la réalisation et/ou gestion des trois projets et quartiers durables étudiés.

Deuxièmement, les investigations de terrain auprès des habitants sont la partie la plus conséquente de notre travail de terrain. C'est pour elle que la démarche emboîtée a principalement été pensée, car c'est dans l'échange avec les habitants que les différents verrous se montrent persistants. Pour ce faire, trois méthodes combinées ont été successivement déployées. La première est celle des entretiens ouverts courts (une trentaine par quartier). Ces entretiens, comprenant une vingtaine de questions, étaient organisés en trois sousparties : signalétique de l'habitant; qualification du 
lieu habité, du quartier et des pratiques; qualification des ambiances et des paysages des quartiers; " définition » des termes « ambiance ", « paysage », « quartier durable ». L'objectif était d'identifier les mots utilisés par les habitants pour parler du sensible, et leurs significations. De plus, ces entretiens constituaient également une première approche pour obtenir des informations sur les quartiers.

Toutefois, il nous a semblé que les différents entretiens auprès des habitants gagneraient à être conduits en parallèle d'autres dispositifs d'observation, mobilisant d'autres moyens d'expression que la parole ou, au moins, différemment. Ainsi, avonsnous mis en place des «parcours multisensoriels » inspirés des parcours commentés (Thibaud, 2001). La particularité de cette méthode est qu'elle apporte des informations prises sur le vif, dans l'action, tout en faisant dans une moindre mesure appel à la mémoire, notamment sensorielle. Elle conjugue « l'être au monde (avec) le faire en cours " (Thomas, 2007, p. 112). Cette méthode a été conçue pour apporter des éléments tout aussi bien sur la question même des rapports sensibles aux territoires de vie que sur d'autres sujets, tels que l'histoire du quartier ou encore les pratiques quotidiennes. Le déroulement des «parcours multisensoriels » s'est fait comme suit : 1. Signalétique des personnes participantes; 2. Dessin sur carte des limites du quartier; 3. Représentation sur une carte des éléments importants, notamment sensoriels, du quartier et préparation de l'itinéraire; 4. Parcours à proprement parlé avec focus sur certains lieux si nécessaire; 5. Questions supplémentaires sur l'appréciation du quartier, les paysages multisensoriels et la durabilité.

Enfin, concernant toujours les investigations auprès des habitants, une dernière approche, plus nouvelle et moins stabilisée dans le corpus des méthodes de ce champ, vise à répondre au besoin de multiplication des langages et des supports d'expression des expériences sensibles. Nous l'avons qualifiée de «baluchons multisensoriels » (figure 1). Cette méthode originale s'inspire en partie des récits mis en place par Augoyard (2001) et des différentes méthodes avec appareils photos mises en place dans le cadre de recherches en paysage (Luginbühl, 1989; Michelin, 1998; Bigando, 2006) ou encore en urbanisme (Lelli, 2003; Gwiazdzinski, 2006). Elle est aussi largement inspirée des outils utilisés par les concepteurs lors de « diagnostics sensibles »

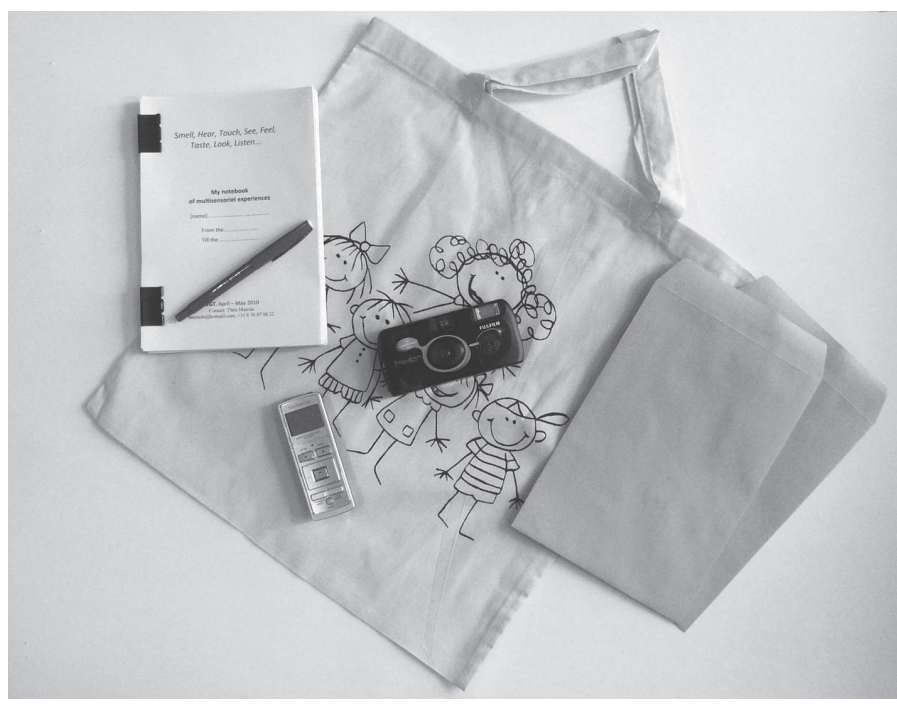

Figure 1 : Un baluchon multisensoriel - T. Manola, 2012 A multisensory pouch - T. Manola, 2012

ou d'«immersions dans le site» (ex. prise de photo, récolte d'objets, prise de notes), croisée à une rigueur et approche plus proches des habitudes scientifiques.

Sous le vocable de "baluchons multisensoriels », il s'agit d'assigner, comme cela peut être fait dans le cadre des «enquêtes transport »: un carnet, dans l'esprit d'un carnet de voyage avec des plans du quartier incorporés, dans lequel les habitants peuvent inscrire, au cours d'une semaine, tous leurs « sentir » et « ressentir » au contact de leurs pratiques et cheminements quotidiens. Pour pallier aux possibles difficultés liées à l'écriture et multiplier les différentes mises en langage, les supports d'expression y sont multipliés : un appareil photo jetable, un enregistreur numérique de poche, ainsi que plusieurs enveloppes pour recueillir des « objets». Ce dispositif peut être qualifié de relativement « impersonnel » car le chercheur n'est pas présent au moment de la réalisation. Cela semble permettre une plus grande aisance dans l'expression. Il se veut «plaisant » de par les différents supports et la liberté laissée pour rendre l'expérience plus active. De plus, cette méthode apporte d'autres éléments d'information, puisqu'elle conjugue à la fois la mémoire des sens et des sensations sur le vif. Le recours à des moyens d'expression autres que la parole (photos, dessins, enregistrements de sons, récolte d'objets) peut non seulement apporter des informations différentes 
mais aussi contrecarrer ce problème récurrent de la mise en langage de l'expérience sensible.

Enfin, soulignons que les deux grands temps méthodologiques exposés se nourrissent mutuellement : les investigations de terrain auprès des habitants alimentent les diagnostics et ces derniers permettent une prise de contact plus aisée avec les habitants. Cependant, par commodité, nous pouvons considérer ces deux temps, au moins intellectuellement, séparables. Dans la suite de cet article, nous focaliserons notre attention uniquement sur les investigations de terrain auprès des habitants des quartiers étudiés, car c'est dans ce domaine que les difficultés et les questions restent les plus prégnantes. Mais avant que d'aller plus loin, un détour par une présentation succincte des trois quartiers d'application de notre méthode s'impose.

\section{Une démarche méthodologique appli- quée à des terrains d'étude spécifiques : des quartiers dits durables}

Nos réflexions et méthodologies ont été appliquées à trois quartiers dits "durables ». Ce choix est dicté par les caractéristiques paysagères souvent attribuées à ces quartiers, qui peuvent en faire des contextes propices à la saisie des rapports multisensoriels aux territoires de vie, par le biais du paysage. Les quartiers étudiés sont les suivants.

Le Wilhelmina Gasthuis Terrein (WGT) à Amsterdam (Pays-Bas) est un quartier habité depuis 25 ans. Il résulte d'une opération de renouvellement urbain d'un ancien site hospitalier qui a vu le jour dans les années 1980 suite à des contestations locales quant à la démolition de l'hôpital qui a conduit à l'occupation illégale du site (figure 2 planche I).

Augustenborg, à Malmö (Suède), est une opération de « réhabilitation écologique » d'un quartier d'habitat social de 3000 habitants, très stigmatisé. Le projet a visé la requalification esthétique et écologique du quartier, mais aussi le respect des modes de vie spécifiques des populations et leur implication dans un processus qui est en mouvement perpétuel (figure 3 - planche II).

Le quartier de Bo01, à Malmö (Suède), fut, quant à lui, créé à l'occasion de l'exposition européenne de l'habitat en 2001. Son esthétique architecturale et paysagère fut très «travaillée ». Ce quartier se caractérise notamment par une mise en avant des écotechnologies, un emplacement en bord de mer, ainsi qu'une extension déjà engagée (figure 4 planche III).

Choisis tout autant pour leur qualité paysagère que pour la diversité des contextes sociaux qu'ils abritent, ces trois quartiers européens se sont vus appliqués la même démarche méthodologique (supra). Ici, il s'agira de revenir sur les apports et limites des investigations de terrain déployés auprès des habitants pour aider à l'expression et à la compréhension de leur(s) paysage(s) multisensoriel(s), tout en accordant une importance aux précisions pratiques de leur mise en place.

\section{RETOUR CRITIQUE SUR LA DÉMARCHE MÉTHODOLOGIQUE MISE EN PLACE}

\section{La créativité méthodologique à l'épreuve du terrain : l'évolutivité de la démarche en question}

Comme nous allons le présenter, la démarche méthodologique pensée initialement a évolué de diverses manières, tout au long du processus des investigations de terrain, afin de s'adapter aux besoins de notre sujet de travail et aux difficultés rencontrées sur le terrain.

\section{À la recherche d'un équilibre entre les apports de chaque méthode}

La première variable qui a évolué au contact du terrain est le poids relatif des différentes méthodes. $\mathrm{Si}$, initialement, les entretiens courts étaient privilégiés, un rééquilibrage qualitatif a été opéré au fur et à mesure de l'avancement de notre travail, tel que le nombre d'entretiens a été revu à la baisse sans pour autant que les informations fournies en soient affectées. Concrètement, la première phase d'investigations in situ s'est soldée par une forte homogénéité et récurrence des réponses données dans le cadre des entretiens ouverts courts réalisés avec les habitants. Il a alors été décidé de réaliser, non pas 50, mais environ 30 entretiens auprès d'habitants de chaque quartier.

Notons ici que le fait d'avoir deux étapes séparées d'investigations de terrain, ainsi que trois terrains d'études, a particulièrement facilité cette adapta- 
tion. Ainsi, la pré-analyse des premiers entretiens faite, nous avons aussi décidé de rééquilibrer la place des deux autres méthodes d'investigation auprès des habitants : prévoyant initialement 15 parcours multisensoriels et 3 baluchons multisensoriels par quartier, leur nombre a été stabilisé à une dizaine pour chacune d'entre elles. Mais au-delà de ce rééquilibrage dans l'importance respective des méthodes déployées, c'est également le protocole des baluchons qui s'est vu évoluer.

\section{Des carnets, coffrets, ou baluchons multisensoriels : que choisir?}

La démarche a aussi évolué dans son contenu méthodologique. Initialement, seul un « carnet multisensoriel » devait être donné aux habitants participants afin qu'ils puissent le compléter pendant une semaine et y inscrire leurs vécus sensoriels quotidiens. Suite aux premières investigations de terrain, c'est-à-dire les entretiens ouverts courts, et une reconsidération de nos priorités, nous avons choisi de proposer d'autres supports que l'écrit : appareil photo, enregistreur sonore, possibilité de récolter des objets.

Cette seconde méthode avait été conçue sous la forme d'une boîte pour se dénommer « coffret multisensoriel ». Or, au moment de la préparation du terrain et de la mise en place pratique des protocoles d'enquête, nous avons choisi de recourir à un sac/baluchon pour administrer cette méthode afin que les participants puissent se l'approprier et se déplacer plus aisément avec. Ainsi, les « carnets multisensoriels » sont-ils devenus des «baluchons multisensoriels ».

Ces évolutions méthodologiques sont facilitées et même insufflées par une démarche méthodologique emboîtée, invitant à chaque étape de la recherche à reposer les termes du travail d'un point de vue méthodologique. Ainsi, évolutivité se décline ici avec emboîtement.

Au final, dans le cadre de ces investigations de terrain, et après les différents ajustements opérés, nous avons mené auprès d'habitants de WGT, Augustenborg, et BO01 : 100 entretiens ouverts courts, 27 parcours et 23 baluchons. Notre démarche exploratoire ne visait pas la représentativité des points de vue mais la diversité des vécus et expériences sensibles. Si les participants n'ont pas été « choisis » dans une logique de représentativité, ils n'en restent pas moins relativement représentatifs des compositions socioculturelles des quartiers étudiés, en termes de situations socioprofessionnelles, de types de logements occupés et de statuts d'occupation, de genre et d'âge notamment. Enfin, si les participants aux entretiens et aux parcours pouvaient habiter les quartiers de manière permanente ou occasionnelle, en tant que visiteurs ou travailleurs, les participants aux baluchons étaient tous des habitants permanents des quartiers. Quels sont alors les apports respectifs à chacune des méthodes déployées, justifiant leur emboîtement et les résultats plus généraux du travail mené?

\section{Une démarche méthodologique : des méthodes aux apports complémentaires pour mieux saisir le sensible situé}

\section{Des discours multiples}

Au vu du corpus recueilli, les trois méthodes présentées se complètent bien, apportant chacune un type d'informations différentes sur les rapports du sujet/habitant à autrui et à son environnement quotidien (tableau 1). En effet, l'entretien favorise un discours assez conventionnel, faisant appel à des représentations communes, comme l'illustre cet extrait d'un habitant de WGT: «(Landscape is) what I see by my window. »(W-E33) $)^{9}$. Le parcours, lui, permet une certaine spontanéité du discours, liée au moment présent mais aussi à sa durée et, à la fois, la possibilité de se rappeler au fur et à mesure des éléments qui auraient pu être oubliés : "This part is the swimming area, you have to try swimming!!! The water is like 19-20 so it's ok. When you go down and the weather is good, you can jump, and there are plenty of people...»(B-P2). L'habitant étant plus impliqué par et dans cette méthode, il oscille entre informations générales, vécus personnels et expériences sensibles. Quant au baluchon, réalisé sur une semaine, la personne prend le temps et raconte une situation, décrit un lieu, parle de ses sentiments. Par le récit, elle construit son propre paysage, invitant l'autre à entrer dans l'intimité sen-

\footnotetext{
9. Consigne de lecture du codage : la première lettre correspond au quartier tels que $\mathrm{W}=\mathrm{WGT}, \mathrm{B}=\mathrm{Bo} 01, \mathrm{~A}=$ Augustenborg; la seconde lettre, après le tiret, correspond à la méthode tels que $\mathrm{E}=$ entretien, $\mathrm{P}=$ parcours, $\mathrm{B}=$ baluchon; et le numéro correspond à l'ordre de la passation sur le terrain. Pour exemple, W-E33 est le $33^{\mathrm{e}}$ entretien mené à WGT.
} 


\begin{tabular}{|c|c|c|c|}
\hline & Entretiens & Parcours & Baluchons \\
\hline $\begin{array}{l}\text { Sensorialité des } \\
\text { paysages }\end{array}$ & $\begin{array}{l}\text { Discours conventionnel } \\
\text { Représentations collectives }\end{array}$ & $\begin{array}{c}\text { Discours plus spontané } \\
\text { Représentations collectives et } \\
\text { personnelles }\end{array}$ & $\begin{array}{c}\text { Récit } \\
\text { Expériences sensorielles de lieux } \\
\text { Avis, jugements }\end{array}$ \\
\hline $\begin{array}{l}\text { Rapport au type de } \\
\text { méthode }\end{array}$ & Distante & Confiante & Intime \\
\hline Niveau d'engagement & $\begin{array}{c}\text { Faible } \\
\text { De } 10 \text { à } 30 \text { min }\end{array}$ & $\begin{array}{c}\text { Moyen } \\
\text { De } 30 \text { min à } 1 \text { h30 }\end{array}$ & $\begin{array}{l}\text { Conséquent } \\
\text { l semaine }\end{array}$ \\
\hline Stabilité & +++ & ++ & - \\
\hline
\end{tabular}

Tableau 1 : Types de discours, niveaux d'engagement et stabilité des méthodes (D’après Geisler et Manola, 2011)

Methods, discourses, levels of commitment required, level of stability of the method (According to Geisler and Manola, 2011)

sible de l'être. Et lorsque les mots lui manquent, elle peut utiliser un autre media comme le dessin, la photo ou l'enregistrement : "Walked back home but since Elyra didn't get to swim like we promised her we stopped at the water sculptures where she loves to play instead. There we spent at least one hour where she played with sand and water. All her clothes were wet and finally she and another girl run around naked, collecting water from the fountain, carrying it to the hand to make mud. Or just sitting in the hole of the fountain pretending it was a bath $\Rightarrow>$ recorded sounds of them playing in the water and took photos » (B-B6).

Outre les types de discours mobilisés (conventionnel, spontané, récit) par chaque méthode, les trois qui ont été appliquées ont des apports complémentaires en ce qui concerne le type de paysages et les sens mobilisés.

\section{Des lieux et territoires habités ${ }^{10}$ pour révéler le sensible par le(s) paysage(s)}

Il ressort de notre analyse que trois types de paysages sont mobilisés dans les discours habitants. Ils sont associés à des formes révélatrices du sensible : des lieux et des territoires. Tout espace, lieu ou territoire, véritablement habité, au sens d'Heidegger, peut entrer dans cette typologie. Toutefois, des distinctions restent à opérer entre les différents lieux et territoires, et leurs niveaux ou manières d'être habités. Cette typologie n'est en rien étanche : les paysages et leurs habiter se « superposent » souvent pour une même personne voire pour plusieurs, et

10. La notion d'habiter évoque « des relations [...] dans lesquelles la Terre est éprouvée comme base » (Dardel, 1952, p. 47) ou encore la « spatialité des acteurs individuels» (Lévy et Lussault, 2003, p. 440), laquelle se caractérise «par une forte interactivité entre ceux-ci et l'espace dans lequel ils évoluent. La notion donne la part belle au rôle de l'individu, du langage, des réalités idéelles. » (Lévy et Lussault, 2003, p. 442). chacun d'entre eux renvoie à une des méthodes utilisées. Trois types de paysages émergent ainsi.

Tout d'abord, des «paysages symboliques » ou «paysages vitrines », construits par et/ou pour autrui, sont évoqués. Ce sont des lieux publics, connus voire reconnus, jugés comme symboliques du quartier et qui en font parfois l'image : ils sont alors habités par les représentations qu’ils véhiculent et qu'ils construisent. Evoqués dans la majorité des entretiens et décrits dans certains parcours multisensoriels, ce sont des lieux représentatifs du quartier certes pour leurs habitants mais aussi pour les personnes de l' " extérieur », compris ou non dans les limites « officielles » du quartier. Par exemple, à WGT, c'est l'entrée de l'ancien hôpital et plus largement la partie réhabilitée de l'ancien hôpital ; à Bo01, la promenade au bord de mer et la tour; à Augustenborg, les entrées fleuries.

À ces paysages "symboliques/vitrines ", s'ajoutent des «paysages communs » de la société locale spatialisée. Il s'agit des lieux et territoires de la vie quotidienne du quartier, souvent publics et qui sont pratiqués, souvent à plusieurs, par la majorité des habitants du quartier. Ils font l'objet d'un rapport affectif ou sont simplement habités de manière quotidienne, pour des raisons fonctionnelles et pratiques. Ce faisant, ils sont principalement décrits lors des parcours, et, dans une moindre mesure, lors des entretiens et dans le cadre de baluchons. Pour exemple, les squares paysagers entre les maisons, à l'intérieur du quartier, ou encore la promenade en bord de mer constituent les paysages communs de Bo01.

Enfin, les «paysages intimes » du soi spatialisé sont des lieux et territoires «intimes », privés ou semipublics, appropriés par des petits groupes et dans lesquels la personne peut se sentir seule au monde. Ils sont habités par des pratiques personnelles quo- 
tidiennes et font le plus souvent l'objet d'un rapport affectif fort. Souvent associés à la «maison » au sens de Bachelard, ils sont liés à des expériences sensorielles plus personnelles. La description de ces lieux et territoires est faite soit par association de sens, soit par l'évocation d'un moment sensoriel et/ou sensible géographiquement attaché. C'est pourquoi ces «paysages intimes » sont parfois décrits lors des parcours, mais plus encore racontés dans les baluchons.

Au-delà de ces trois types de paysages et des rapports affectifs respectifs qui y sont attachés, nos trois méthodes se révèlent également complémentaires par les enseignements qu'elles apportent sur l'appréhension et la compréhension de la (multi) sensorialité.

\section{Des apports sensoriels différenciés}

Conformément aux objectifs fixés, plus nous avançons dans la démarche, plus les méthodes renseignent la sensorialité (tableau 3). Ainsi, si dans le cadre des entretiens, les références aux rapports sensoriels se font rares et d'autant plus en ce qui concerne les sens les moins «nobles » (odorat, toucher, goût), nous constatons une augmentation des occurrences relatives aux rapports sensoriels dans le cadre des parcours et des baluchons (tableau 2).

En résumé, les trois méthodes se complètent, certes en partie par leur protocole et par leurs objectifs, mais surtout par leurs apports en termes de connaissances du terrain, des vécus, des sensibles, et d'autre part, en termes d'informations recueillies : discours, paysages décrits et racontés, rapports sensoriels exprimés (tableau 3).
Avec l'appui des parcours et dans une moindre mesure des baluchons, les entretiens ouverts courts ont permis de connaître les mots utilisés par les habitants pour parler du sensible spatialisé : paysage, ambiance mais aussi nature, architecture et art. Les parcours multisensoriels ont conduit à mieux appréhender les expériences partagées, notamment sensibles, c'est-à-dire les expériences personnelles communes à plusieurs personnes du quartier ou les expériences partagées, vécues à plusieurs. Par le croisement avec les entretiens et dans une moindre mesure les baluchons, ils ont permis de connaître les formes spatiales et sociales qui révèlent le sensible. Les baluchons ont, quant à eux, permis de dévoiler les expériences sensibles intimes et, dans leur croisement avec les parcours, les sens du sensible et le sens caché derrière chaque sens. Enfin et surtout, le croisement de tous ces éléments permet d'entrevoir ce qu'est le sensible. Pour aller plus loin dans notre retour critique sur la méthodologie mise en place, nous proposons alors de dresser une première évaluation quant à l'expérimentation des baluchons multisensoriels.

\section{Les baluchons multisensoriels : une méthode aux apports singuliers}

Dans le cadre de notre démarche, nous avons proposé la mise en place d'une méthode inédite et élaborée pour répondre à un besoin précis : libérer ou du moins faciliter l'expression du sensible. Dans le cadre de l'analyse des 23 baluchons réalisés, il nous a semblé important de porter une attention particulière à leurs avantages et forces, manques et imperfections.

\begin{tabular}{|c|c|c|c|c|c|c|}
\hline & & Vue & Ouïe & Odorat & Toucher & Goût \\
\hline \multirow{3}{*}{ WGT } & Entretien (43) & 4 & 3 & 3 & 1 & - \\
\hline & Parcours (9) & 39 & 44 & 27 & 13 & 7 \\
\hline & Baluchon (8) & 46 & 32 & 33 & 12 & 4 \\
\hline \multirow{3}{*}{ Bo0l } & Entretien (30) & 7 & 4 & 3 & 8 & - \\
\hline & Parcours (10) & 38 & 23 & 16 & 33 & 3 \\
\hline & Baluchon (7) & 43 & 53 & 24 & 53 & 19 \\
\hline \multirow{3}{*}{ Augustenborg } & Entretien (29) & 2 & 4 & 1 & - & - \\
\hline & Parcours (10) & 18 & 19 & 16 & 8 & 5 \\
\hline & Baluchon (8) & 55 & 54 & 17 & 21 & 15 \\
\hline Total & & 251 & 236 & 140 & 140 & 62 \\
\hline
\end{tabular}

Tableau 2 : Nombre d'occurrence par quartier, sens et méthode - Manola, 2012 Number of occurrences per quarter, senses and method-Manola, 2012 


\begin{tabular}{|c|c|c|c|}
\hline Méthode & Entretiens & Parcours & Baluchons \\
\hline Discours & $\begin{array}{l}\text { Discours conventionnel } \\
\text { Représentations collectives }\end{array}$ & $\begin{array}{c}\text { Discours plus spontané } \\
\text { Représentations collectives et } \\
\text { vécus personnelles }\end{array}$ & $\begin{array}{c}\text { Récit } \\
\text { Expériences sensorielles } \\
\text { Avis, jugements }\end{array}$ \\
\hline Paysages & symboliques & communs & intimes \\
\hline Sens & $\begin{array}{c}\text { Vue - } 13 \\
\text { Ouïe - } 11 \\
\text { Odorat - } 7 \\
\text { Toucher - } 9 \\
\text { Goût - } 0\end{array}$ & $\begin{array}{c}\text { Vue - } 95 \\
\text { Ouïe - } 86 \\
\text { Odorat - } 59 \\
\text { Toucher - } 54 \\
\text { Goût - } 15\end{array}$ & $\begin{array}{c}\text { Vue - } 144 \\
\text { Ouïe - } 139 \\
\text { Odorat - } 74 \\
\text { Toucher - } 86 \\
\text { Goût - } 38\end{array}$ \\
\hline
\end{tabular}

Tableau 3 : La démarche emboitée et la construction des paysages multisensoriels - Manola, 2012

The methological approach and the construction of multi-sensory landscapes - Manola, 2012

Nous proposons ici de voir en détail leurs spécificités méthodologiques en présentant succinctement dans un premier temps les supports d'expression utilisés et les difficultés persistantes, puis, dans un second temps, les apports spécifiques de cette méthode.

\section{Le baluchon multisensoriel : prometteur mais perfectible}

Les baluchons récupérés après avoir été confiés une semaine aux participants, notre préoccupation première était de connaître : la nature et le nombre de moyens utilisés pour exprimer les rapports sensibles aux territoires de vie, le nombre de jours et les moments choisis pour s'y livrer, le niveau d'information atteint. Autrement dit, notre première interrogation était de savoir si les baluchons avaient bien rempli leur fonction de faire parler des sens, et notamment de ceux les moins exprimés dans les autres méthodes : l'odorat, le toucher, le goût.

Il en ressort que les baluchons répondent dans les grands traits à leur objectif : par un discours plus personnalisé, le corpus recueilli apporte des informations sur des lieux intimes, évoque directement les sens et sensations, relate l'expérience sensible des individus par rapport à un moment $t$ mais aussi à plusieurs moments. Il est à penser que le fait de proposer des moyens d'expression multiples a ainsi, comme attendu, participé à une relative libération du sensible. Qu'il s'agisse de baluchons tenus une seule journée ou une semaine entière, les rapports multisensoriels sont bien présents dans les discours.

Cependant, nous avons souhaité poser aussi un regard plus critique sur cette méthode nouvelle. Pour ce faire, nous avons mis en place une double évaluation : d'une part par les habitants et, d'autre part, par nos propres soins, suite à l'analyse du corpus. Comme nous allons le montrer, cette première expérimentation, certes déjà très satisfaisante, laisse entrevoir de possibles améliorations.

\section{Les baluchons vus par les habitants : exigeants mais plaisants}

Lors de l'administration des baluchons multisensoriels, nous avons décidé de mettre en place une évaluation sur le vif de la méthode, pressentant qu'un entretien post-baluchon pourrait être riche de sens et d'enseignements. Lors de cette rapide évaluation, nous avons adressé les groupes de questions suivantes aux 15 habitants directement revus pour récupérer le baluchon :

- Comment avez-vous trouvé cette approche/ méthode? Avez-vous eu des difficultés? Y avez-vous trouvé un intérêt particulier?

- Est-ce que cette expérience vous a permis de «voir» autrement votre quartier? Avez-vous fait attention à des choses qui passent inaperçues d'habitude?

- Est-ce que vous auriez aimé avoir d'autres supports? Est-ce que vous auriez aimé avoir plus/moins de consignes? Pour quel support était-ce le plus compliqué? Pourquoi?

De manière générale, un intérêt vif a été déclaré par toutes les personnes pour avoir un retour sur la recherche, ainsi qu'un grand intérêt pour la méthode elle-même, laquelle a permis aux habitants de (re) découvrir leur quartier : "It changed the way I looked at the neighbourhood... really, because I experience it everyday so I don't think about those things... » 
(W-B4); "It changed a little bit things... I look more in details and it made me more vigilant... looking about things... searching them...» (B-B1).

Concernant les moyens offerts par la méthode pour saisir les rapports sensoriels, la majorité des personnes a été largement satisfaite par les supports proposés, même si certaines n'ont pas utilisé la totalité d'entre eux.

Ce qui ressort de manière assez forte est que les baluchons multisensoriels comportent une notion de plaisir et de découverte dans leur réalisation, à l'instar d'un jeu sensoriel : "It's very relaxing actually, doing nothing besides recording and taking pictures and notes...» (A-B5); «I enjoyed it a lot. »(B-B7); "I'm not used to think by all the senses, but it was amusing! It was really great to experience it! » (A-B4).

Suite à l'analyse du corpus issu des baluchons et le retour des habitants, certaines difficultés semblent toutefois persister.

\section{Des difficultés persistantes}

Pour quatre des participants, les deux plus grandes "failles » de cette méthode sont le manque de temps des personnes, comme cela est souvent évoqué en sciences humaines et sociales, et d'autre part, la non «naturalité » des expériences. Les habitants n'ont par exemple pas l'habitude de prendre des photos ou des enregistrements d'objets ou des moments qui provoquent des réactions sensorielles et/ou sensibles : "Touching... it wasn't... of course you touch your bike but... I don't know but I didn't go touch staff, because I don't do it usually... I thing it's interesting but I felt a little bit forced to search things and when you search for it it's like not my normal usual sensation...»(W-B2). À ces deux premières difficultés, une autre s'ajoute et concerne l'instabilité de la méthode (tableau 4). Ainsi, la richesse des informations est très dépendante certes du temps

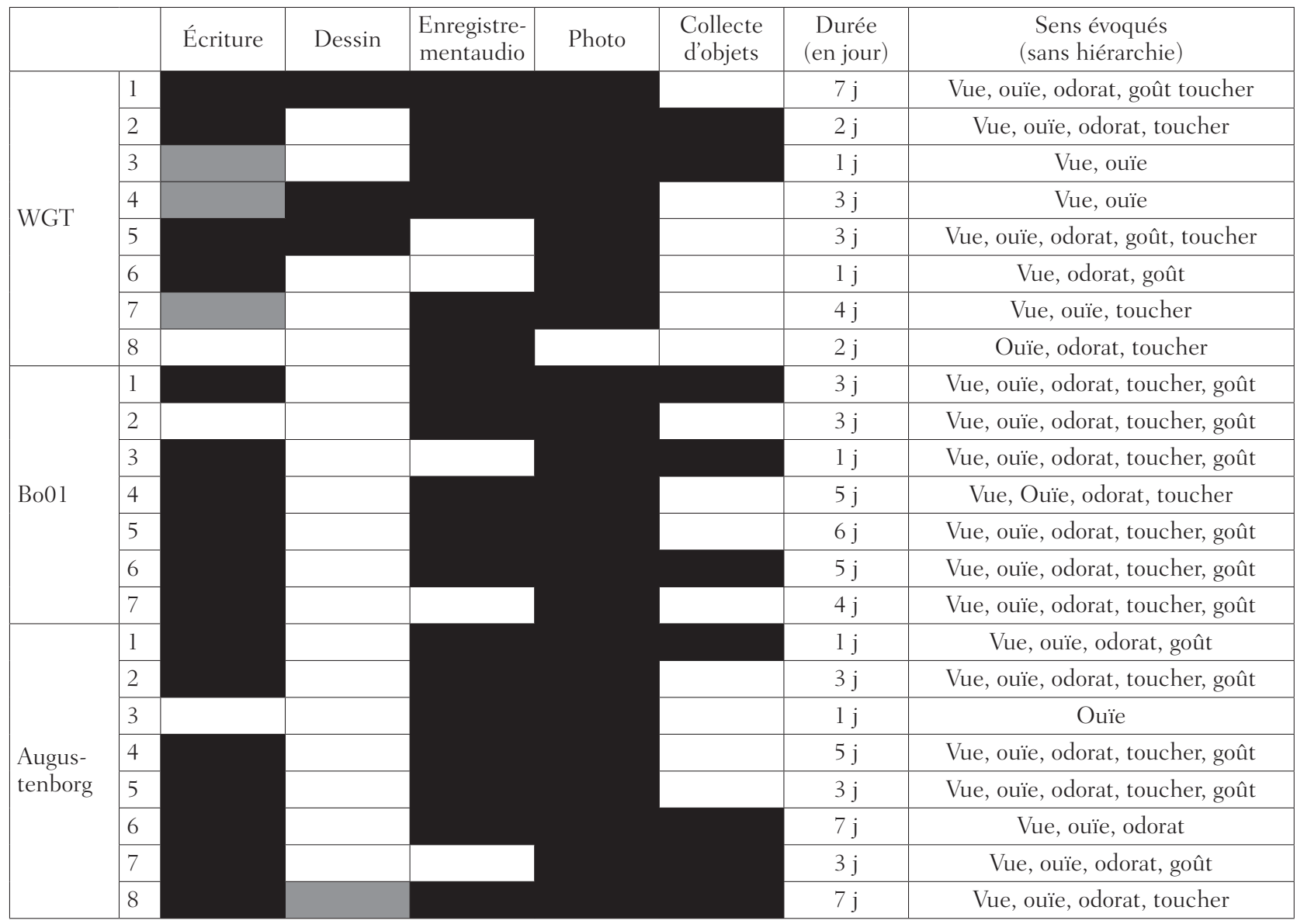

Tableau 4 : Durée, moyens d'expressions utilisés et sens mobilisés, dans le cadre des baluchons - Manola, 2012 Multi-sensory pouches: Duration, means of expression used and senses mobilized - Manola, 2012 
consacré par l'habitant, mais également de sa facilité et de son plaisir à écrire, à utiliser un appareil photo, un enregistreur sonore...

Cependant, comme nous le verrons par la suite, les baluchons peuvent être vus comme une méthode fort prometteuse : ils fournissent de précieuses informations que ni l'entretien ni le parcours multisensoriel n'apportent. Tout d'abord, cela concerne le rapport qui se révèle fort et intime entre l'habitant et le baluchon : l'habitant n'est plus seulement un « objet » d'observation qui sert une recherche, mais devient peu à peu un acteur principal, à part entière. Plus encore, les informations recueillies concernent des moments, des lieux et/ou des actions auxquels

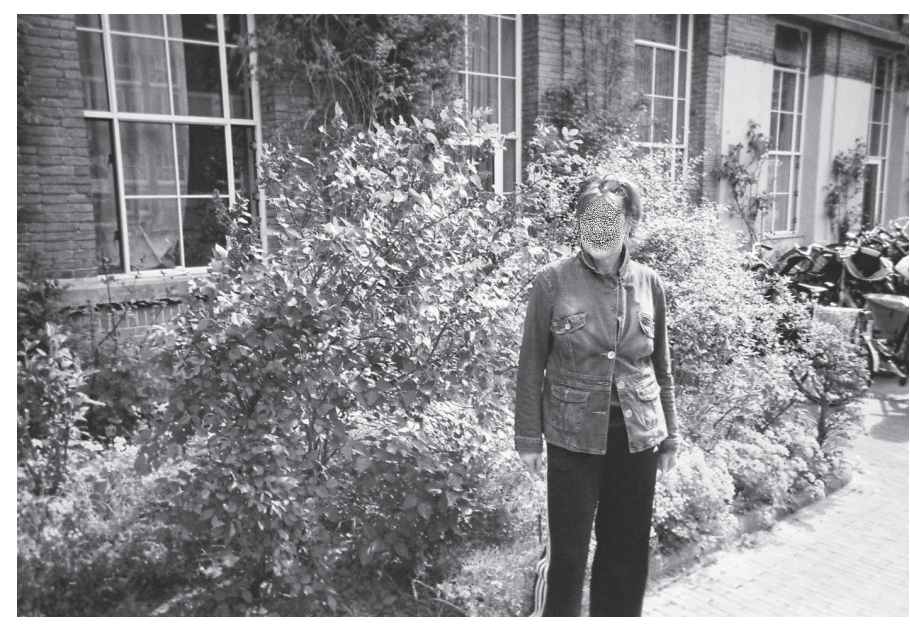

Figure 5: «A picture of my neighbor Selma in our garden... ( (W-B4) - Extrait de baluchon multisensoriel

Extract of multisensory pouch

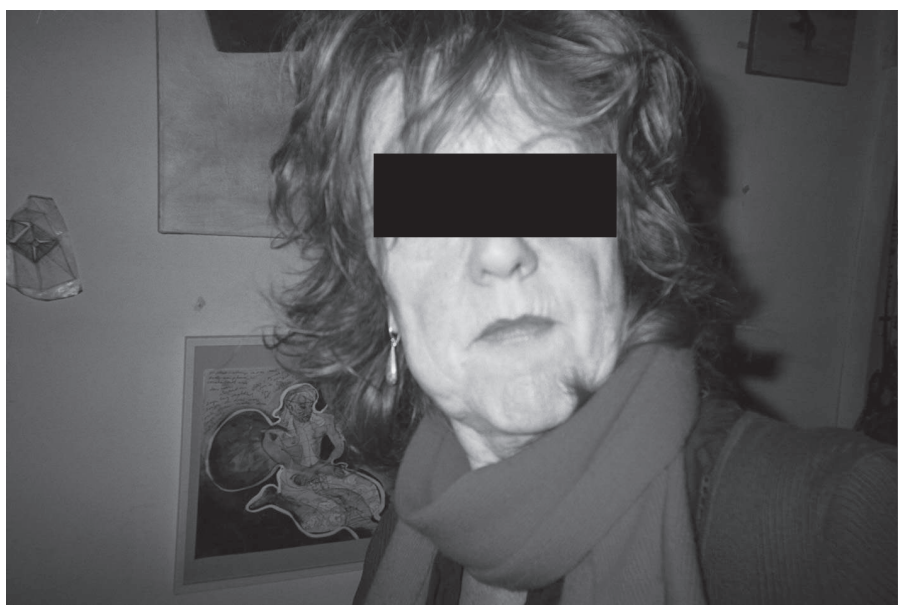

Figure 6 : «(Photo) In my studio - wall-painting + self portrait. » (W-B5) Extrait de baluchon multisensoriel

Extract of multisensory pouch un enquêteur n'a habituellement pas accès. Comme nous allons maintenant l'expliciter, leur expression, souvent lié à un mode discursif plus poétique, exprime bien souvent des accolements sensoriels, des associations sensorielles, du vécu multisensoriel.

\section{Les baluchons : une méthode intimiste, les habitants acteurs premiers de la méthode}

Une des spécificités des baluchons est l'investissement bien personnel de leurs participants, lesquels nous partagent tout autant leur intimité que celle de leur entourage. Par exemple, les habitants se sont souvent photographiés eux-mêmes ou ont pris pour modèles leurs amis, voisins ou enfants (figures 5 et 6). De même, il arrive que les personnes enregistrent leurs pensées : "I look really weird talking to this thing... People look at me... there is a family that seems to have just come back from the beach..." (A-B5). Et, se prêtant au jeu, les participants n'ont pas hésité également à interviewer d'autres personnes, à nous interpeller directement, ou encore à enregistrer leurs enfants, amis et voisins, et ce notamment dans des moments privés de la vie de tous les jours.

Dans ce registre, le journal multisensoriel a parfois une fonction de journal intime, à travers lequel les habitants nous racontent leur journée, ce qu'ils ont fait, ressenti, etc., au point d'oublier semble-t-il que l'appareil photo ou l'enregistreur finira dans les mains d'un « étranger».

Or, de toute évidence, ces actes sont bien conscients : plusieurs personnes nous ont laissé des messages personnels soit enregistrés, soit écrits ou dessinés dans le cahier ou sur des post-it ajoutés (figure 7). La place et le rôle des habitants dans la méthode sont importants et sont perçus comme tels. Les habitants sont acteurs de leur histoire et le revendiquent d'une certaine manière. De ce fait, il n'y a plus de distanciation par rapport à la méthode : les participants se "parlent » à eux-mêmes plutôt qu'à un enquêteur, tout en étant conscients de sa présence future. La méthode d'enquête, pleinement appropriée, c'est eux! Ceci est aussi montré par des investissements parfois très importants des baluchons : en témoignent de nombreux dessins et soulignages (figure 8), mais également l'attrait poétique du style adopté, comme nous allons le voir. 
«Un journal multisensoriel est aussi un journal intime» (Extraits de discours).

1. Going over the garden from our house.

2. Walking over the pebles (sound).

3. Stopping at the water sculpture (photos and sound). Spider webs everywhere.

4. Café, chocolate and cookies (sound and photos) 5. Sailing contest (photo and sounds).

6. Going to a friends house in the near by Dochan and from there to Slottstaden to play with our kids.

7. Walking back to Vastra Hamnen along the canal then beside the harbour, home.

8. Photo: they are tearing down the old building in front of ICA Maxi, planning to build new houses. Looking forward to see who they turn out.

9. Going for a sunset walk and to listen to one of the month's sunset concerts down by Titanic. On the way down to the sea I go through my favourites small alleys and passages. »(B-B6).

"We had my father in law and his wife over for the day. Walked from our house down to spot restaurant where we bought pizza, which we took with us down to Ribberborgs beach. We had lunch on the beach, planed to go to a swim but the rain stopped us. Walked back to Bo01. When we crossed the bridge (BR1) the sun came out again, and we stopped to watch kids jumping from the bridge into the water in the harbour. They put a big sign "danger, don't jump-dive here". But it hasn't stopped young boys from all over town to come there to jump. An accident waiting to happen I think. » (B-B4).

\section{Les baluchons laissent place à des discours poétiques et (multi)sensoriels}

Comme entrevu plus haut, le discours des baluchons est plus spontané, plus proche du récit. Il laisse aussi place à un discours bien plus poétique, voire à des poèmes proprement dits pour les plus inspirés.

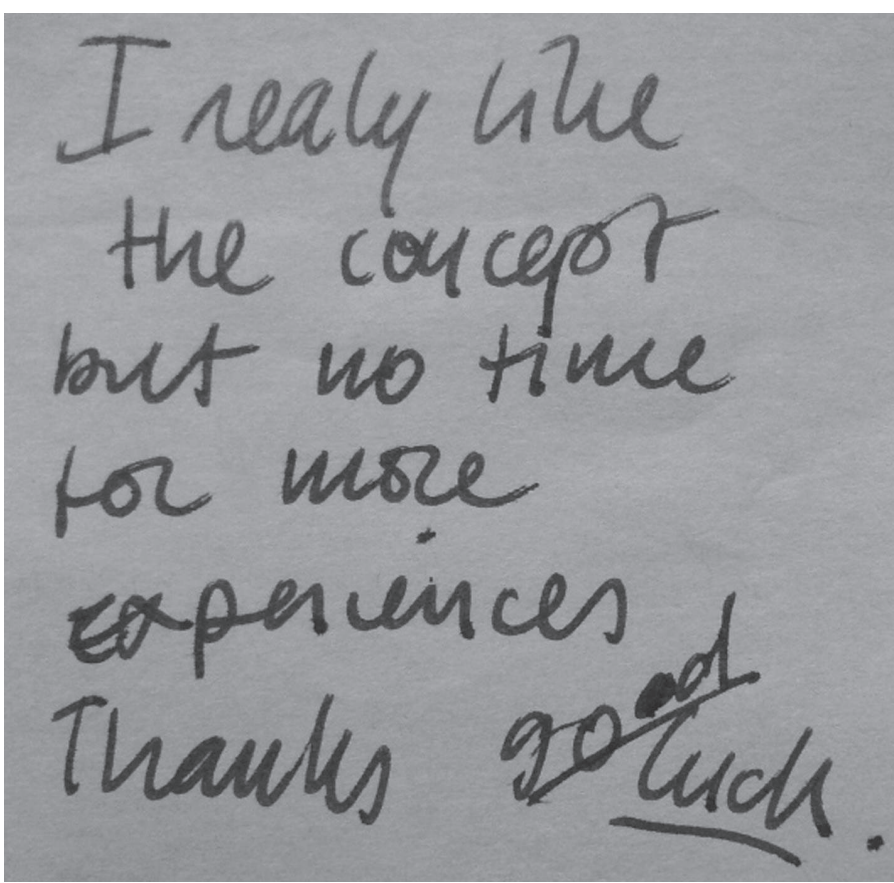

Figure 7 : Post-it collé sur le baluchon W-B3 Post-it on the pouch W-B3

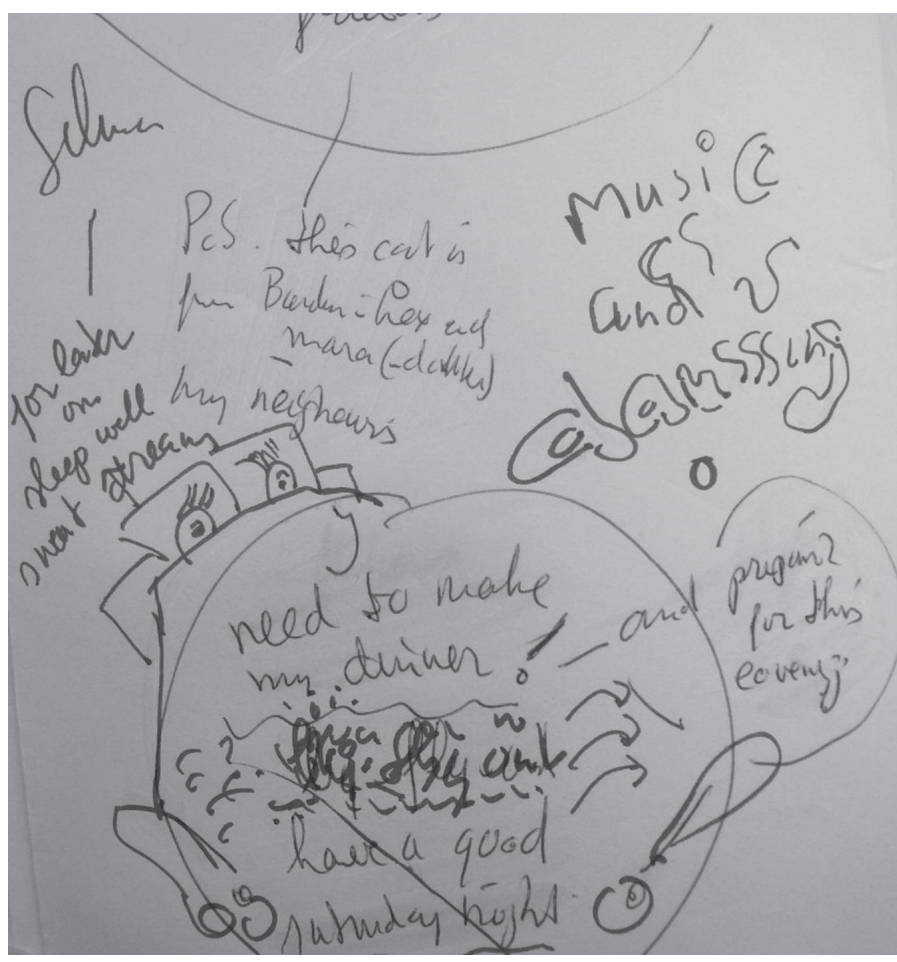

Figure 8 : Messages personnels issus du baluchon W-B5 Personnal messages from the pouch $W-B 5$ 
Discours poétiques et poèmes - extraits des discours habitants

«Almost $35^{\circ} \mathrm{C}$. Walking down the stairs towards the sea, just a short stroll away from home. Small pebbles and rough concrete makes me jure I won't slip. Over to the more soft warmth of the wooden part. The sun scorches. A slight jump, the feeling of equilibrium in mid-air and the plunge and semi-chilly engulfing salty water. On the underwater steps the intense green sea-weed entangles my toes. Lift up my 9 month old daughter Freja and smiles to her lavender sun-warm skin with it's sweet odor and mind boggling softness. » (B-B1) «Saturday 24 of April 2010

Early in the morning

It is getting on the edge

Of dark and light

Birds are singing

Slowly the sun is coming

Braking through »

(W-B5)

«Black, mushy, fat soil around my hands, under my fingernails, in my sweating pores. The dry and slender bamboo, the delicate and scent-oozing jasmine and rose now stands in new pots. The arduous job gets an excellent grand finale with a yeast sweet Hoegaarten wessbeer so cold the bottle immediately is dew-strewn. Ah, the splendor of the first greedy gulp as the beer rushes down the throat. » (B-B1)

Une autre grande spécificité des baluchons par rapport à notre thématique de travail est qu'ils traitent directement et quasiment de manière exclusive des rapports sensoriels. Les manières de raconter les vécus sensoriels prennent trois formes :

- des accumulations d'expériences sensorielles. Pour illustration simplifiée : « j’ai vu un arbre, j’ai entendu des oiseaux, j’ai goûté une pomme du jardin »;

- des associations sensorielles. Pour illustration simplifiée : «j'ai vu et entendu des enfants jouer »;

- ou encore des récits de moments sensoriels, où l'on raconte une expérience et la ponctue de nos impressions, sentiments, souvenirs, etc. Pour illustration simplifiée : "J'ai mangé une tarte aux pommes; elle sentait tellement bon; ça m’a rappelé l'odeur du four et les mains dans la pâte quand je cuisinais avec ma mère... »

Les sentiments, jugements et affects se libèrent ainsi par la multisensorialité, apportée ici principalement dans notre démarche méthodologique par les baluchons. Les baluchons semblent ainsi être aussi une méthode pertinente pour aborder de manière indirecte les rapports affectifs à la ville (Martouzet, 2007 ; Feildel, 2010) par exemple.

Que nous dévoilent alors très concrètement ces baluchons?

\section{Baluchons : avoir accès à l'inaccessible}

Le dernier apport spécifique des baluchons est de révéler des lieux et des moments auxquels nous avons rarement accès. Comme vu, les paysages intimes et privés apparaissent dans les discours obtenus grâce aux baluchons. En fait, en dépit de la consigne attachée au journal du baluchon, les habitants ont assez souvent préféré nous relater non pas des parcours effectués en tant que tels, mais plutôt des lieux et territoires, à travers leurs expériences sensibles et leurs habiter. Somme toute, ce sont bien des paysages multisensoriels qu'ont permis de révéler cette nouvelle méthode. En comparaison aux autres méthodes, et notamment aux parcours, les habitants y citent et y racontent leurs expériences sensorielles sur un nombre moindre de lieux et de territoires. Mais, il s'agit des lieux et territoires les plus pratiqués, aimés, habités, intimes... Ces lieux et territoires se concentrent surtout dans les parties véritablement vécues du quartier, ceux que les habitants investissent tous les jours pour des raisons pratiques (ex. parking à vélos), affectives (ex. leur jardin, leur balcon, leur salon), avec une forte concentration sur les lieux, territoires et paysages en lien direct avec la maison de la personne détentrice du baluchon.

Une autre spécificité des baluchons est de nous donner accès à des expériences sensorielles produites à des moments de la journée qui ne sont pas habituellement traités dans le cadre d'enquêtes: très tôt le matin, tard la nuit. Il s'agit ici d'un apport important car l'évolution des paysages dans le temps, notamment à l'échelle du rythme journalier, semble avoir une grande importance selon les discours habitants et participe de manière évidente au ressentir général d'un lieu ou d'un territoire. La méthode 
Horaires habituellement inaccessibles - extraits des discours habitants

"Evening: another bat flying in the half dash sky. Lovely flower smell (place B). Starry, bright night sky! (dessin but and evening) ( (W-B 1)

"Early in the morning. (4 o'clock) the birds are singing. Slowly the sun comes through the clouds. » (W-B5)

"Half five in the morning I recorded the sound of the sub-evident chirping of birds, moreover, it was quite quiet. You feel a great calm. » (A-B2)

"I'm in the heavy night now, so maybe I do not hear/smell so much as I usually do. » (A-B8)

pourrait alors se prêter à d'autres thématiques de recherche, notamment relatives à la prise en compte du temps dans l'action urbaine.

\section{Conclusions}

La fin de la mise en place de notre démarche méthodologique sur le terrain date de juillet 2010. Depuis, l'ensemble du corpus recueilli a été analysé au bénéfice de notre travail doctoral et d'une autre recherche susmentionnée. Surtout, nous sommes aujourd'hui en mesure de porter un regard plus critique sur notre démarche et sur ses retombées. Avant de discuter les adaptations et évolutions possibles de la démarche méthodologique, nous souhaitons insister ici sur trois points qui nous semblent essentiels et qui constituent des enseignements méthodologiques centraux de notre travail.

Premièrement, une démarche méthodologique est par essence le fruit d'un processus dans le temps, à savoir le temps de la recherche. Elle est alors un produit évolutif et adaptable. Ce sont bien ces deux éléments qui ont permis à notre démarche de répondre à son objectif, à savoir : saisir les rapports multisensoriels à l'espace et le sensible.

Deuxièmement, la démarche est certes pensée de manière emboîtée en amont grâce à un travail théorique et des retours d'autres expériences, mais fait également ses preuves par les enseignements respectifs à chaque méthode. Dans notre cas, l'emboitement des entretiens ouverts courts, des parcours multisensoriels et des baluchons multisensoriels, réalisés auprès d'habitants, semble réussi tant par la complémentarité des discours que des résultats obtenus.

Aussi, afin de réussir notre entreprise et dépasser les contraintes préalablement identifiées, nous ne nous sommes pas cantonnée aux méthodologies existantes. Nous avons développé une autre méthodologie, propre aux besoins de notre sujet de travail, le paysage multisensoriel. Cette méthodologie, baptisée «baluchons multisensoriels », semble satisfaire en grande partie sa fonction première : faire parler des sens et du sensible. In fine, nous pouvons affirmer que, grâce à une méthode adéquate, les habitants peuvent s'exprimer sur leurs paysages multisensoriels et les renseigner en fonction des habiter qui les structurent.

Or, malgré la réussite de notre démarche méthodologique en vue de saisir et de comprendre le sensible situé, certaines limites existent.

D’un point de vue général d'abord, la démarche utilisée pourrait être plus riche en méthodes compréhensives, lesquelles permettraient de traiter, dans le cadre d'une démarche emboîtée, sens et signification au même niveau. Nous pourrions alors imaginer de remplacer les entretiens courts par des entretiens ouverts longs; ce qui permettrait non seulement de saisir le sensible mais aussi de l'expliquer plus avant. Dans cette même optique, filmer les entretiens, comme cela a déjà pu être expérimenté en ethnographie, et les analyser en mobilisant alors peut-être d'autres savoirs et disciplines, pourrait aider à mieux comprendre le sensible.

Deuxièmement, il serait probablement judicieux d'inverser l'ordre des méthodes : placé avant un parcours et/ou un entretien, le baluchon multisensoriel pourrait avoir le rôle de "réveil sensoriel » quand il est réalisé avec une même personne. Le corpus du baluchon pré-analysé, un parcours et/ ou un entretien pourrait alors suivre afin que le chercheur puisse mieux comprendre le contenu du baluchon tout en le complétant par d'autres informations, moins centrées sur l'intimité spatiale et sensorielle de l'être. Ce réajustement permettrait de travailler et d'échanger avec des personnes ayant déjà « réfléchi » sur un temps long sur leurs rapports sensoriels et faciliterait certainement les échanges sinon permettrait d'aboutir à des résultats plus qualitatifs encore car plus en profondeur.

En ce qui concerne spécifiquement les baluchons, la méthode présente un fort intérêt par sa finesse, 
l'intimité qu'elle instaure et le détail des informations recueillies. Maintenant que les baluchons ont été testés comme méthode individuelle et que nous commençons à avoir un nombre d'informations conséquent sur la thématique du sensible (situé spatialement et socialement) et sa médiation - le paysage multisensoriel -, ne pourrions-nous pas imaginer une autre déclinaison des baluchons vers une méthode collective? Cette alternative pourrait être utile afin de pallier aux difficultés des individus, et donner naissance à un discours préalablement négocié entre les participants. Aussi, cela nous permettrait de dépasser les limites des méthodes individuelles qui ne permettent pas « de comprendre comment la perception façonne et est façonnée par les relations que les individus entretiennent les uns avec les autres, comment elle peut devenir le support d'une action collective " (Colon, 2008 , p. 8). Ces «baluchons collectifs », dont les modalités pourraient se nourrir d'autres retours d'expériences pourraient être utilisés en complément ou non de baluchons individuels. Ils seraient envisagés soit comme une initiation à l'expression des rapports sensoriels et sensibles et donc au début d'une démarche emboîtée, soit comme étape de validation collective des acquis issus de méthodes individuelles, voire pour l'édification de propositions et préconisations en aval d'une démarche embồtée. Outre ces considérations méthodologiques, les baluchons collectifs sembleraient particulièrement adaptés comme outils d'aide à des situations et territoires conflictuels par exemple ou encore des projets en cours.

Enfin, se pose la question de l'utilité de cette méthode, en dehors des apports spécifiques à la connaissance scientifique. Que faire du matériau récolté? Comment peut-il aider à une prise en compte des rapports multisensoriels et sensibles dans l'action urbaine et plus spécifiquement dans le cadre de projets urbains ? L'ultime étape de notre démarche, non présentée dans cet article, a consisté en la formalisation d'un diagnostic mixte pour un des trois quartiers étudiés, WGT à Amsterdam. Il s'est agi de mettre en place un système cartographique, sur un seul support cartographique, qui reprend : les diagnostics urbanistiques (mobilité, fonctions du bâti, qualification des espaces publics); les diagnostics sensibles de la chercheure (différentes modalités sensorielles et analyse des poten- tialités du terrain); diagnostics issus de l'analyse du corpus recueilli auprès des habitants du quartier (différentes modalités sensorielles et qualification générale des lieux). Une double carte finale croise les différents résultats et exprime ainsi le caractère habité/inhabité du quartier (Manola, 2012) ${ }^{11}$. Par ce système cartographique, nous avons souhaité proposer un type de support qui peut servir tout aussi bien comme système d'analyse sensible voire de (pré) projet à un territoire et de ses potentialités, mais qui peut aussi être approprié par tout un chacun par son langage graphique, son apparence proche d'un site internet, ses possibilités d'usage. L'objectif d'un tel système cartographique n'est pas seulement d'exister. Il s'agit en effet de poursuivre ce travail en confrontant ce produit aux habitants du quartier afin de pouvoir imaginer à terme un outil d'implication de la totalité des acteurs intéressés, sur l'avenir d'un territoire qui serait en projet ou ferait l'objet de débats.

Cette démarche méthodologique ainsi que la formalisation de ses résultats constituent un des premiers pas à franchir afin de considérer les rapports sensoriels et sensibles des habitants avec leur(s) ville(s). Il s'agit d'une étape nécessaire pour une véritable considération sensible de l'urbain et envisager une ville de demain plus « humaine » sinon plus habitable.

\section{Remerciements}

Cet article fait suite au workshop "Sentir et ressentir la ville", organisé par A. Madœuf et D. Martouzet dans le cadre du $10^{\circ}$ congrès international de la SIEF, "People Make Places: Ways of feeling the World". Tous nos remerciements vont aux organisateurs $d u$ workshop et aux participants. Nous remercions également vivement les relecteurs, qui ont participé à l'amélioration de ce texte, en particulier Nathalie Gourlot pour ses relectures critiques.

11. [http://www.preview.pa-th.com/thea_manola/]. 


\section{Bibliographie}

Audas N., Martouzet D., 2008. Saisir l'affectif urbain. Proposition originale par la cartographie de réactivation des discours, dans Actes du colloque Penser la ville. Approches comparatives, accessible sur : [http://halshs.archives-ouvertes. fr/halshs-00380553/en].

Augoyard J.-F., 2001. La conduite de récit, dans Grosjean M., Thibauld J.-P. (dir.), L'espace urbain en méthodes, Editions Parenthèse, p. 173-196.

Balez S., 2000. L'observation des ambiances olfactives en milieu urbain, dans Mattei M.-F., Pumain D. (éd.), Données urbaines, ${ }^{\circ} 3$, Paris, Anthropos, Economica, p. 427-435.

BÉDARD M. (dir.), 2009. Le paysage comme projet politique, Presses de l'université du Québec, 330 p.

Berque A., 2000. Ecoumène, introduction à l'étude des milieux humains, Belin, Paris, coll. « Mappemonde», 272 p.

Besse J.-M., 2009. Le goût du monde. Exercices de paysage, Paris, Actes Sud/ENSP, coll. «Paysage », 228 p.

Bigando E., 2006. La sensibilité au paysage ordinaire des habitants de la grande périphérie bordelaise (communes du Médoc et de la Basse Vallée de l'Isle), thèse de doctorat en géographie, université de Bordeaux 3, 490 p.

Blanc N. (dir.), Bridier S., Choen M., Glatron S., GreSILlON L., 2004. Des paysages pour vivre la ville de demain. Entre visible et invisible, UMR LADYSS/CNRS, 256 p.

Callon M., Lascoumes P., Barthes Y., 2001. Agir dans un monde incertain. Essai sur la démocratie technique, Paris, Seuil, 358 p.

Colon P.-L., 2008. Du sensible au politique : vers une nouvelle approche de l'environnement sonore, Communication au Colloque Espaces de vie, espaces-enjeux : entre investissements ordinaires et mobilisations politiques organisé par RESO (UMR 6590) et le CRAPE (UMR 6051), université Rennes 2 et IEP de Rennes, 12 p., accessible sur : [www.eso. cnrs.fr/TELECHARGEMENTS/colloques/rennes_11_08/ Colon_Paul_Louis.pdf].

Corbin A., 1986. Le miasme et la jonquille, l'odorat et l'imaginaire sociale XVIII ${ }^{e}$-XIX ${ }^{e}$ siècle, Paris, Flammarion, 334 p.

Dardel E., 1952. L'Homme et la Terre: nature de la réalité géographique, CTHS, $199 \mathrm{p}$.

Dubois D., 2006. Les «mots » et les catégories cognitives du sensible : des rapports problématiques. Des couleurs, des odeurs et des bruits, Cahiers du LCPE, $\mathrm{n}^{\circ} 7$, septembre, p. 19-38.

Faburel G. (resp. scient.), Manola T., Geisler E., Davodeau H., Tribout S., 2011. Les quartiers durables: moyens de saisir la portée opérationnelle et la faisabilité méthodologique du paysage multisensoriel?, Rapport de recherche, Lab'Urba, 158 p.

Faburel G., Manola T. (coord.), Brenon L., Lévy L., Gourlot N., Grenier A., Charre S., Leservoisier S., Marcou M., Tong Canh T., Benoît G., 2007. Le sensible en action. Le vécu de l'environnement comme objet d'aide à la décision, Rapport de recherche, CRETEIL, 84 p., (non publié).

Feildel B., 2010. Espaces et projets à l'épreuve des affects. Pour une reconnaissance du rapport affectif à l'espace dans les pra- tiques d'aménagement et d'urbanisme. Thèse de doctorat en aménagement de l'espace et urbanisme, université François Rabelais de Tours, $670 \mathrm{p}$.

Geisler E., Manola T., 2011. Les paysages de Kronsberg à Hanovre : approches sonore et multisensorielle, Projets de paysage, $\mathrm{n}^{\circ}$ 6, 23 p., accessible sur : [http://www.projetsdepaysage.fr/fr/les_paysages_de_kronsberg_a_hanovre_ approches_sonore_et_multisensorielle].

Grésillon L., 2010. Sentir Paris. Bien-être et matérialité des lieux, Quae, Paris, 191 p.

GréSillon L., 2010. De l'espace de qualité à celui du bienêtre : une question d'appropriation sensorielle?, dans FlEURET S. (dir.), Espaces, qualité de vie et bien-être, Actes du colloque international d'Angers des 23 et 24 septembre 2004 « Peut-on prétendre à des Espaces de Qualité et de Bien-Être? », Presses de l'université d'Angers, p. 34-43.

Grosjean M., Thibauld J.-P. (dir.), 2001. L'espace urbain en méthodes, Parenthèse, $214 \mathrm{p}$.

Gwiazdzinski L. 2006. Traversées nocturnes, dans Le Floc'H M., Mission repérage. Un élu un artiste, Editions L'entretemps, p. 241-242.

LeLli L., 2003. La photographie de paysage comme outil de mobilisation des acteurs pour un projet de territoire, dans Debardieux B., Lardon S. (dir.), Figures du projet territorial, Paris, Édition de L'Aube/Datar, coll. « Bibliothèque des territoires », p. 183-191.

LÉvy J., Lussault M., 2003. Dictionnaire de la géographie et de l'espace des sociétés, Paris, Belin, 1034 p.

LuginbüHL Y., 1989. Paysages élitaires et paysages ordinaires, Ethnologie française, vol. XIX, n³ 3, p. 227-236.

Luginbühl Y., 2001. La demande sociale de paysage, Rapport pour le Conseil National du Paysage, Ministère de l'Aménagement du Territoire et de l'Environnement, 21 p., (non publié).

LuginbüHL Y., 2005. Le paysage pour penser le bien-être?, dans Fleuret S. (dir.), Espaces, qualité de vie et bien-être, Presses de l'université d'Angers, Actes du colloque international d'Angers des 23 et 24 septembre 2004 « Peut-on prétendre à des Espaces de Qualité et de Bien-Être? », p. 225-242.

Manola T., 2012. Conditions et apports du paysage multisensoriel pour une approche sensible de l'urbain. Mise à l'éprenve théorique, méthodologique et opérationnelle dans 3 quartiers dits durables européens: WGT, Bo01, Augustenborg. Thèse de doctorat en Urbanisme, aménagement et politiques urbaines, université Paris-Est Créteil Val-de-Marne (UPEC), 646 p.

Manola T., Geisler E., 2012. Du paysage à l'ambiance : le paysage multisensoriel. Propositions théoriques pour une action urbaine sensible, dans Thibaud J.-P., Siret D. (dir.), Ambiances en acte(s), p. 677-682.

Marot S., 1995. L'alternative du paysage, Le Visiteur, ${ }^{\circ} 1$, p. $54-81$.

Martouzet D., 2007. Le rapport affectif à la ville : premiers résultats, dans Paquot T., Lussault M., Younès C. (eds.), Habiter, le propre de l'humain : villes, territoires et philosophie, Paris, La Découverte, p. 171-191. 
Michelin Y., 1998. Des appareils photo jetables au service d'un projet de développement : représentations paysagères et stratégies d'acteurs locaux de la montagne thiernoise, Cybergeo : European journal of geography, Politique, culture, représentations, Document 65, accessible sur : [http://cybergeo.revues.org/index $5351 . \mathrm{html}$.

Paquot T., 2010. L'urbanisme c'est notre affaire!, Atalande, $176 \mathrm{p}$.

Sansot P., 2004. Poétique de la ville, Paris, Payot, 625 p.

Terrasson D., 2007. Introduction, dans Berlan-Darqué M., Luginbühl Y., Terrasson D. (dir.), Paysages : de la connaissance à l'action, Quae, p. 11-14.

Thibaud J.-P., Balez S., Boyer N., Couic M.-C., Fiori S., Saraiva M., Thomas R., Tixier N., 1998. Comment obser- ver une ambiance? Ambiances architecturales et urbaines, Les Cahiers de la recherche architecturale, $\mathrm{n}^{\circ}$ 42/43, p. 77-91.

Thibault J.-P., 2001. La méthode des parcours commentés, dans Grosjean M., Thibauld J.-P. (dir.), L'espace urbain en méthodes, Parenthèse, p. 89-99.

Thomas R., 2007. Quand le pas fait corps et sens avec l'espace. Aspects sensibles et expressifs de la marche en ville, Cybergeo, accessible sur: [www.cybergeo.eu/index4304.html].

Vouligny E., Domon G., 2006. La compréhension des valorisations paysagères. Vers une nouvelle méthode d'analyse des paysages de l'ordinaire, Chaire en paysage et environnement - Université de Montréal, 17 p., (non publié). 